\title{
Characterization of Supersonic Radiation Diffusion Waves
}

A. S. Moore ${ }^{\mathrm{a}, 1, *}$, T. M. Guymer ${ }^{\mathrm{a}}$, J. Morton ${ }^{\mathrm{a}}$, B. Williams ${ }^{\mathrm{a}}$, J. L. Kline ${ }^{\mathrm{b}}$, N. Bazin ${ }^{\mathrm{a}}$, C. Bentley ${ }^{\mathrm{a}}, \mathrm{S}$. Allan ${ }^{\mathrm{a}}$, K. Brent ${ }^{\mathrm{a}}$, A. J. Comley ${ }^{\mathrm{a}}$, K. Flippo ${ }^{\mathrm{b}}$, J. Cowan ${ }^{\mathrm{b}}$, J. Martin Taccetti ${ }^{\mathrm{b}}$, K. Mussack ${ }^{\mathrm{b}}$, D. W. Schmidt ${ }^{\mathrm{b}}$, C. E. Hamilton ${ }^{\mathrm{b}}$, K.

Obrey $^{\mathrm{b}}$, N. E. Lanier ${ }^{\mathrm{b}}$, J. Workman $^{\mathrm{b}}$, R. Mark Stevenson ${ }^{\mathrm{a}}$

${ }^{a} A W E$ Aldermaston, Reading, RG7 4PR, United Kingdom

${ }^{b}$ Los Alamos National Loboratory, Los Alamos, NM 87545 USA

\begin{abstract}
Supersonic and diffusive radiation flow is an important test problem for the radiative transfer models used in radiationhydrodynamics computer codes owing to solutions being accessible via analytic and numeric methods. We present experimental results with which to compare these solutions by studying supersonic and diffusive flow in the laboratory. We present results of higher-accuracy experiments than previously possible studying radiation flow through up to 7 high-temperature mean free paths of low-density, chlorine-doped polystyrene foam and silicon dioxide aerogel contained by an Au tube. Measurements of the heat front position and absolute measurements of the x-ray emission arrival at the end of the tube are used to test numerical and analytical models. We find excellent absolute agreement with simulations provided that the opacity and equation of state are adjusted within expected uncertainties; analytical models provide a good phenomenological match to measurements but are not in quantitative agreement due to their limited scope.
\end{abstract}

Keywords: radiative transfer, diffusion, plasmas, shock waves, experiment, supersonic

PACS: 71.35.-y, 71.35.Lk, 71.36.+c

\section{Introduction}

Phenomena in which the flux of radiation emitted by a heated body $\left(\mathbf{S}_{r}=\sigma T^{4}\right)$ exceeds the conduction of energy by heated material $\left(\varepsilon \rho C_{s}\right)$ occur in a range of exotic plasmas from the laboratory to astrophysical scale. Here $\sigma$ is the Stefan-Boltzmann constant $\left[\mathrm{W} / \mathrm{m}^{2} / \mathrm{K}^{4}\right], T$ is temperature $[K], \varepsilon$ is the specific internal energy $[\mathrm{J} / \mathrm{g}], \rho$ is mass density $\left[\mathrm{g} / \mathrm{m}^{3}\right]$, and $C_{s}$ is the sound speed $[\mathrm{m} / \mathrm{s}]$. In $3 \mathrm{D}$ this is a difficult and complex class of physical problem in which not only is the equation of radiative transfer a function of seven independent variables. While in a simpler 1D planar geometry this reduces to four variables, account must still be taken of the complex dependence of material properties (opacity and $\varepsilon$ ) on the relevant independent variables. Through various approximations analytical solutions to $1 \mathrm{D}$ problems have been found, but owing to the complexity of almost all 2D and 3D problems, solutions are necessarily numerical via computer simulation. In many cases these computer codes are also used to simulate and answer questions about the structure of stars, the behavior of supernovae and closer to home, in the laboratory, the transport of x-ray energy in Inertial Confinement Fusion targets $[1-3]$. As with all simulations it is essential that

\footnotetext{
* Corresponding author.

Email address: alastair.moore@physics.org (A. S. Moore)

${ }^{1}$ Currently at Lawrence Livermore National Laboratory, P.O. Box 808, Livermore, CA 94551-0808 USA
}

they be benchmarked against data and analytic reference problems to verify the numerical procedures.

In this paper we detail the first experimental measurements in a previously inaccessible regime to examine one of the few semi-analytic solutions that does exist in this area - the 'Marshak wave' problem [4-8]. As one of the few problems dealing with radiation transport that has analytic solutions it is often used to provide quantitative validation of numerical results. We generate experimental results to test the applicability of several previously published analytic approximations and fully-integrated radiation hydrocode simulations.

We present results that characterize supersonic and diffusive radiation flow in a highly diffusive regime, using a $310 \mathrm{eV}$ temperature $\mathrm{x}$-ray source to drive a radiation heat front at downstream Mach $(\mathcal{M}=6)$ through more than 6 Rosseland mean free paths. The key to reaching such regimes lies with the laser energy available on the National Ignition Facility (NIF) [9]. Results from previous experiments are summarized in figure 1 . These were typically limited to regimes with velocities of $\mathcal{M}=2-4$ and investigated flow of a lower radiation temperature (120-150 eV) through 3-4 mean free paths [10-13]. In this regime the ionization of material does not lead to an energetically significant re-radiated flux, and thus the experiments were not in a fully diffusive regime. Experiments by Back et al. extended the radiation drive to $\sim 190 \mathrm{eV}$, but were limited by the foam-scale and laser energy[14]. The energy now available on the NIF enables a more constraining class of 


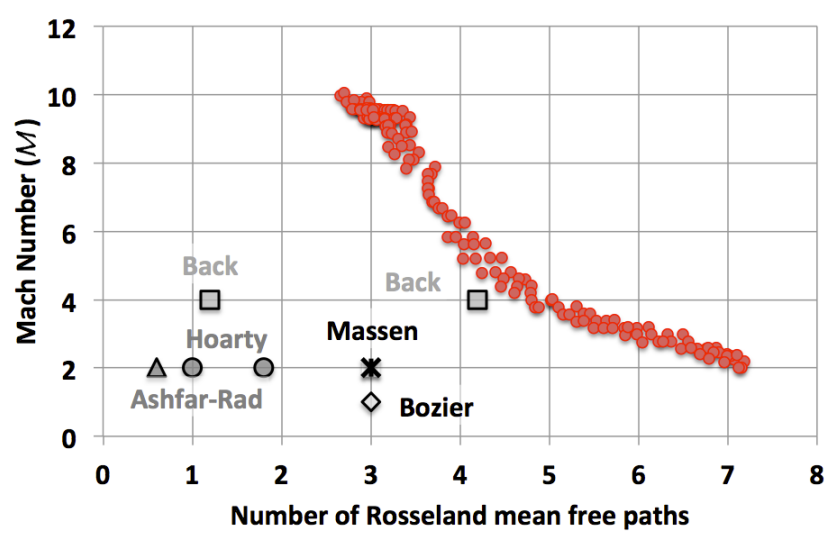

Figure 1: Radiation flow Mach number $\mathcal{M}$ compared to the number of Rosseland mean free paths at the material temperature; Calculations of the Rosseland mean free path vs time for the experiments we present are extracted directly from simulations and are shown by the red points. Previous experiments include[10-14].

experiment using higher, more uniform density foam that is more accurately characterized, and generating a brighter heat front from which more accurate measurements of $\mathrm{x}-$ ray emission are made than was previously possible.

In section 2 we describe the theoretical problem, develop the arguments behind the simple diffusion model from which much of the analytical results described in section 2.1 are developed. In section 2.2 we describe the different numerical approximations that we later apply to compare with the experimental results. The experiment is detailed in section 3.1, with the primary results described in sections 3.2 and 3.3. Finally, the comparison of measurements to the various analytical and numerical solutions are discussed in section 4 .

\section{The General Problem and Solutions}

The 1D problem is typically posed as a cold absorbing slab medium occupying a semi-infinite half-space $[6,15]$. The medium is assumed to be perfectly homogenous and initially at zero temperature with no external radiation sources present. A constant temperature radiation source is applied at the boundary, heating the surface and reducing its opacity. Solutions aim to find the position of the heat-front vs time as it penetrates into the cold medium and the spatial shape of the temperature profile.

$$
\begin{aligned}
\frac{\partial}{\partial t}\left[\rho \varepsilon+\frac{\rho u^{2}}{2}+U_{r}\right]=-\nabla \cdot & {\left[\rho \mathbf{u}\left(\varepsilon+\frac{u^{2}}{2}\right)+\right.} \\
& \left.\left(P_{m}+P_{r}\right) \mathbf{u}+\mathbf{S}_{r}\right]
\end{aligned}
$$

Beginning with the energy equation containing external radiation sources - Eq. (1), several simplifying assumptions are made immediately: (1) that radiation energy density $\left(U_{r}\right)$ and pressure $\left(P_{r}\right)$ are negligible compared to material internal and kinetic energy and pressure
$\left(P_{m}\right)$, and $(2)$ that in the supersonic limit any material motion $(u)$ can be ignored. In Eq. (1) $\rho$ is mass density, $u$ the velocity, $\varepsilon$ the material internal energy, and $\mathbf{S}_{r}$ the radiant energy flux.

In addition to the energy equation we must consider the kinetic equation for the distribution function of photons which describes how radiation energy is transferred into the material. If scattering is ignored, then in the conventional form the equation of transfer of the specific radiation intensity $I(\nu, \Omega, r, t)$ can be written:

$$
\frac{1}{c} \frac{\partial I(\nu)}{\partial t}+\Omega \cdot \nabla I(\nu)=\kappa^{\prime}(\nu)\left(I_{p}(\nu)-I(\nu)\right)
$$

where $\kappa^{\prime}$ is the frequency dependent opacity modified for induced emission according to Kirchoff's law, and $I_{p}(\nu)$ is Plank's function for the equilibrium spectral energy density from a blackbody as a function of frequency $(\nu)$ [16]. $c$ is the speed of light in vacuum.

Without going into the details of the underlying assumptions of the diffusion approximation which are welldescribed by Pomraning and Castor, the basic argument relies on the assumption that the angular dependence of the specific radiation intensity is only very weakly anisotropic and can be represented by the first two terms in a spherical expansion i.e. $I(\nu, \Omega)=I_{0}(\nu) / 4 \pi+3 \Omega . \mathbf{I}_{1}(\nu) / 4 \pi[17,18]$. Pomraning elegantly describes how the angular moment of Eq. (2) reduces to a Fick's law form if the radiation field be treated as quasi steady-state by neglecting the imederivative term $[19,20]$. In this approximation it is simple to identify that $I_{0}(\nu)$ is $c U_{r}(\nu)$ and $\mathbf{I}_{1}(\nu)$ is $\mathbf{S}_{r}(\nu)$ yielding the relationship between the flux and radiation energy density in the diffusion limit:

$$
\mathbf{S}_{r}=-\frac{c}{3 \rho \kappa^{\prime}} \nabla U_{r}
$$

Since the integral of an equilibrium blackbody source over a $2 \pi$ half-space results in the relation $S_{r}=c U_{r} / 4=$ $\sigma T^{4}$ the basic underlying equation for supersonic and diffusive radiation transport can be written:

$$
\rho \frac{\partial}{\partial t} \varepsilon(\rho, T)=-\nabla \cdot\left[\frac{4 \sigma}{3 \rho \kappa} \nabla T^{4}\right]
$$

It is worthy of note that by accessing the supersonic and diffusive regime Eq. (4) demonstrates how the problem is essentially described by four main parameters, $\rho$, $T$, $\varepsilon$, and $\kappa_{R}$. Consequently a carefully designed experiment with a well-diagnosed temperature and density can be used to quantitatively constrain a combination of the material properties. This argument will be developed further by Guymer et al., but even with this caveat results in this regime provide a constraining test for analytic and numerical radiation transport models [21]. 


\subsection{Analytic Approaches}

Marshak originally developed similarity solutions under constant density and pressure conditions and coined the term 'Marshak Wave' [4]. This original work imposed an exponentially increasing boundary temperature to derive the well-known steep shape of the temperature distribution at the heat front, but constant temperature and constant energy solutions are readily accessible [2]. These are all typically accessed by approximating the material opacity as a power-law function of temperature and density $[22,24]$, and give slightly differing variants of the textbook solution for the heat front position $x_{f}$ for a constant temperature boundary condition:

$$
x_{f} \propto \sqrt{\frac{\sigma T^{4} t}{\rho^{2} \kappa_{R} \varepsilon}}
$$

Here $\kappa_{R}$ is the Rosseland mean opacity and $t$ is time.

There are a number of different diffusion-like approximations that have been applied to this problem and which are well summarized by Graziani and so will not be discussed at length here. In particular we draw attention to the work of Pomraning that extends Marshak's original work to generate solutions in which the radiation and material fields are not in equilibrium with the aim that the solution be useful as a reference problem for validating computational models, and Larsen who investigated an asymptotic 1D analysis that included the radiation energy density term neglected above and demonstrated that this causes the radiation front to lag behind that of the Marshak prediction [6, 7].

We devote more detail to the work of Hammer et al. and Hurricane et al. since they are of use in comparing to the experimental data we present herein. Hammer et al. extended Marshak's work in 1D by applying a perturbation technique to the non-linearity of the temperature dependencies that enables diffusive solutions for an arbitrary time-dependent temperature drive [22]. Beginning with the standard power-law approximation for $\kappa_{R}$ and $\varepsilon$ :

$$
\frac{1}{\kappa_{R}}=g T^{\alpha} \rho^{-\lambda}, \quad \varepsilon=f T^{\beta} \rho^{-\mu}
$$

they insert these parameters into Eq. (4), and then introduce dimensionless variables for space, time and $T^{4+\alpha}$, to transform Eq. (4) into a form where it can be approximated by an expansion of the variable $\epsilon=\beta /(4+\alpha)$ which is typically small $(<0.3)$. Following this approach they find solutions of increasing accuracy in $\epsilon\left(\epsilon^{2}, \epsilon^{4}\right.$, etc. $)$, and find good agreement with results from the numerical radiation hydrodynamics code HYDRA [23]. For the results we present in section 3.3, this provides an attractive opportunity to study the effects of the temporal dependence of the temperature source present in the experiments, compared to the other analytical models that build from the impractical constant temperature boundary condition. The heat front position $\left(x_{f}\right)$ as a function of time that results from of the analysis of Hammer et al. and that will be discussed in section 4 - Eq. (25) in ref [22] - is shown in Eq. (7) where $T_{r}(t)$ is the time-dependent source temperature at the boundary.

$$
\begin{aligned}
x_{f}(t)^{2} & =\frac{(2+\epsilon) C T_{r}(t)^{-\epsilon(4+\alpha)}}{(1-\epsilon)} \int T_{r}(t)^{4+\alpha} \mathrm{d} t \\
C & =\frac{16}{(4+\alpha)} \frac{g \sigma}{3 f \rho^{2-\mu+\lambda}}
\end{aligned}
$$

In contrast, Hurricane et al. approach Eq. (4) in a different way noting that away fron the heat front the lefthand side is generally negligible [24]. By forcing a temperature independent opacity approximation to simplify the problem, Eq. (4) reduces to a Laplace equation in $T^{4}$, which is then a more readily approachable eigenvalue problem in multiple dimensions. While this approach has obvious failings, it has the advantage that it enables quantification of the effect of energy loss through arbitrary cartesian boundaries which is a practical reality in these experiments. In cartesian coordinates the effect is to introduce a curvature to the heat front which is described by a cosine-dependence, and an additional drag-term to the on-axis heat front. To best adapt this solution for our experiments would require solving a Laplace equation for $T^{4}$ in cylindrical polar coordinates rather than the cartesian results presented by Hurricane et al.. Results now take the form of a modified Bessel differential equation with solutions of the 1st kind in $r$ modified by a similar eigenvalue problem.

The results from Hurricane et al. that we use in the later analysis are primarily $x_{f}(t)$, and the heat front radius of curvature $\left(R_{c}\right)$ shown in Eq. (8). Here $y$ is the lossy wall boundary in the $2 \mathrm{D}$ cartesian plane of Hurricane et al. analysis, which we set as the effective radius of the tube, and $\mathrm{a}$ is the albedo of the gold tube. $D$ is the modified radiation diffusion constant.

$$
\begin{aligned}
x_{f}(t) & \approx \sqrt{D t}-\frac{y}{12} \sqrt{\frac{2}{e}}\left(\frac{D e t}{2 y^{2}}\right)^{\frac{3}{2}}+\ldots, \\
R_{c} & \approx \frac{y}{\sqrt{e} \cosh ^{-1}\left(\frac{D e t}{2 y^{2}}+1\right)} \\
e & =\frac{3}{4} \rho \kappa_{R} y(1-a) \\
D & =\left(1+\frac{e}{3}\right) \frac{8 \sigma T_{r}^{4}}{3 \kappa_{R} \rho^{2} \varepsilon}
\end{aligned}
$$

Critically for all the models that derive from Eq. (4) it must be appreciated that the Milne boundary condition for this half-space problem and limits the applicability of this class of solution [25]. Castor expresses this in the form: 


$$
t>\frac{(\alpha+4) \varepsilon}{6 \kappa_{R} \sigma T^{4}}
$$

which is essentially the ratio of the energy contained in a layer one Rosseland mean free path thick compared to that entering the layer $\left(\sigma T^{4}\right)$ in time $t ; \alpha$ is the result of the opacity parameterization in Eq. (6).

\subsection{Numerical Approaches}

Four different approximations are typically used in numerical radiation transport calculations to approximately solve the Boltzmann equation [26]: (1) Diffusion, (2) Spherical Harmonics $\left(P_{n}\right),(3)$ Discrete Ordinates $\left(S_{n}\right)$, and (4) Implicit Monte Carlo (IMC).

Diffusion, which is essentially the same as a $P_{1}$ spherical harmonic approximation, is the simplest but most obviously inaccurate approximation failing in optically thin plasmas and where energy density gradients are large. Since the diffusion approximation is a first-order correction to an isotropic radiation field, the obvious next level of complexity is to allow the angular distribution of radiation to be more complex.

$P_{n}$ and $S_{n}$ are strategies to approximate the radiation field numerically by either a spherical harmonic approximation of increasing order $n$ or along $n$ discrete lines-ofsight [27]. By taking an increasing number of moments of the transport equation a solution with increasing accuracy is found. While this can in theory be arbitrarily accurate for increasing $n$, when time-dependence is important wave effects can result in unphysical negative energy densities, however this is not typically the case since the transport in the majority of radiation-hydrodynamics simulations can be treated as steady state. The Discrete Ordinate $\left(S_{n}\right)$ approximation assumes that radiation only travels along a finite number of directions - discretizing the angular distribution of radiation. Due to this discrete nature photons do not reach certain regions that in reality they would. In problems of more than one-dimension this can result in large unphysical spatial distortions in the energy density or so called 'ray effects' [28]. For $n$ infinite, both methods find the exact solution to the transport equation. For both methods, increased computational resources now often allow these solutions to be run for increasing $n$ until convergence is found, at which point the solution is assumed to be 'correct'.

Finally Monte Carlo methods, in particular IMC [29] provides an alternative methodology to $S_{n}$ by simulating photons travelling through the simulation grid at each time step and statistically sampling the photon being scattered or absorbed and how this re-distributing energy in the radiation field. Provided sufficient computational processing power and memory are available, this can also produce very accurate results, however complications can occur at boundaries between high and low opacity materials and unphysical 'teleportation' effects can arise if the computational cell size is larger than the photon mean free path.

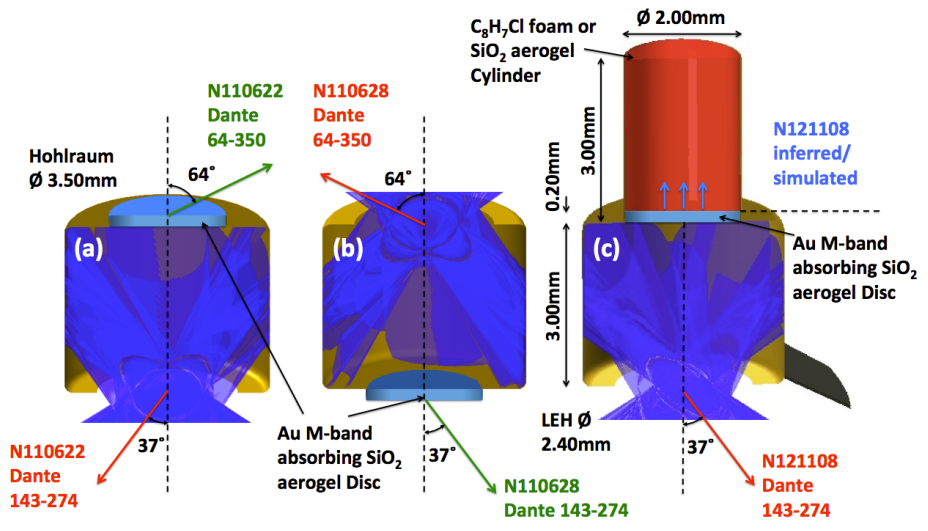

Figure 2: A $3.0 \times 3.5 \mathrm{~mm}$ diameter laser driven half-hohlraum generates a $>300 \mathrm{eV}$ x-ray source. Au M-band radiation is reduced by a thin $200 \mu \mathrm{m}$ disc of $\mathrm{SiO}_{2}$ aerogel before driving a supersonic diffusion wave through $2.8 \mathrm{~mm}$ of chlorinated polystyrene foam or silicon dioxide aerogel. (a) shows the configuration studied in two experiments N110320 with a laser energy of $354.9 \mathrm{~kJ}$ and N110622 with $364.2 \mathrm{~kJ}$, (b) the shows N110628 'flipped' configuration shot with $355.5 \mathrm{~kJ}$ and (c) the configuration to measure the Marshak wave propagation, on N121108 this was shot with $368.1 \mathrm{~kJ}$.

In the same way as with $S_{n}$, IMC is normally run with increasing numbers of particles until the solutions converge.

The convergence of solutions from the IMC and $S_{n}$ methods to the same answer obviously gives a great deal of confidence in that answer, and if numerical solutions to the analytical problems previously described are also in agreement then it could be argued that the models have been validated. However, to ground the different numerical models and their implementation in reality, experimental measurements are required.

\section{Experimental Measurements}

\subsection{Setup}

In these experiments we use a half-hohlraum heated to a temperature of $>350 \mathrm{eV}$ to investigate diffusive $\mathrm{x}$-ray flow in a foam material located opposite the laser entrance hole (LEH) see figure 2. The Au vacuum hohlraum had an inside diameter of $3.5 \mathrm{~mm}$, was $3.0 \mathrm{~mm}$ in length; the $\mathrm{Au}$ was $25 \mu \mathrm{m}$ thick. Eighty $351 \mathrm{~nm}$ laser beams, in three cones, enter the hohlraum through a $2.4 \mathrm{~mm}$ diameter hole. Irradiating the hohlraum at $30^{\circ}, 44.5^{\circ}$ and $50^{\circ}$ from the cylindrical axis the laser beams total $370 \pm 10 \mathrm{~kJ}$ in a 2.5 ns flat-in-time pulse, which is measured on each shot to $1 \sigma= \pm 2.5 \mathrm{~kJ}$. To tailor the x-ray spectrum a $0.2 \mathrm{~mm}$ thick disc of $0.125 \mathrm{~g} / \mathrm{cc} \mathrm{SiO}_{2}$ aerogel was used to preferentially absorb Au M-band x-rays (2.5-3.5keV) and create a more thermal spectrum. The diffusive $\mathrm{x}$-ray flow is studied in a $2.8 \mathrm{~mm}$ long cylinder of Cl-doped $\mathrm{CH}$ foam [31] or $\mathrm{SiO}_{2}$ aerogel placed in contact with the thin aerogel disc and enclosed in a $25 \mu \mathrm{m}$ thick Au tube. A $150 \mu \mathrm{m}$ slot ran the length of the tube to enable measurement of the foam self-emission [30]. 


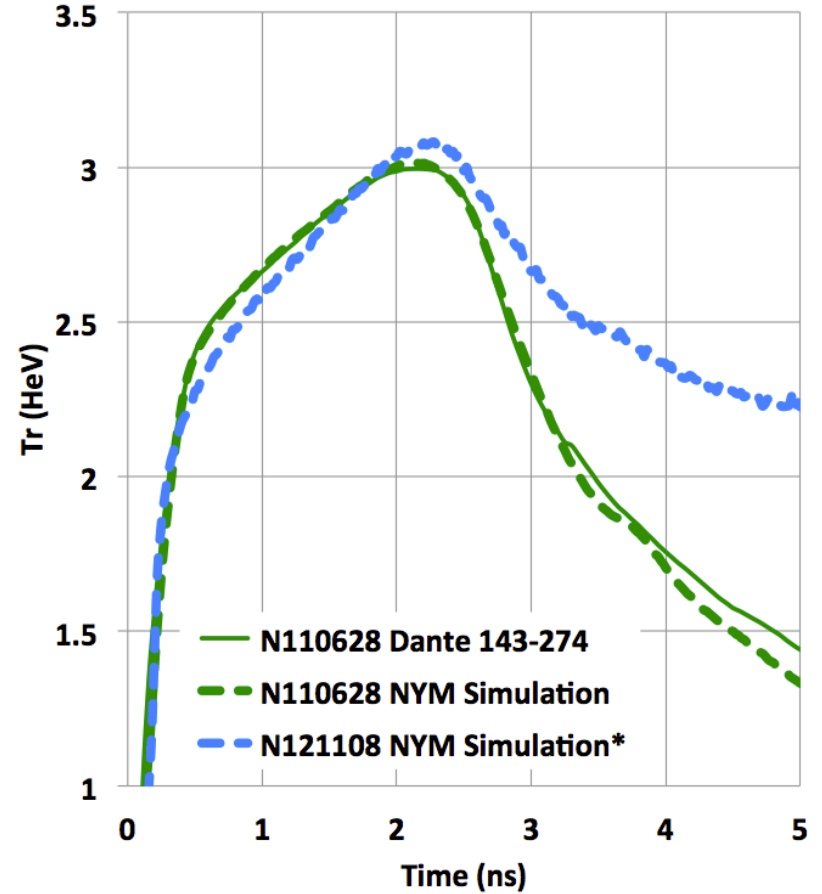

Figure 3: X-ray flux entering foam cylinder measured through $\mathrm{SiO}_{2}$ aerogel disc compared to simulations using the radiation hydrodynamics code NYM and inferred for the supersonic Marshak wave arrival in Table 1 based on the delivered laser energy.

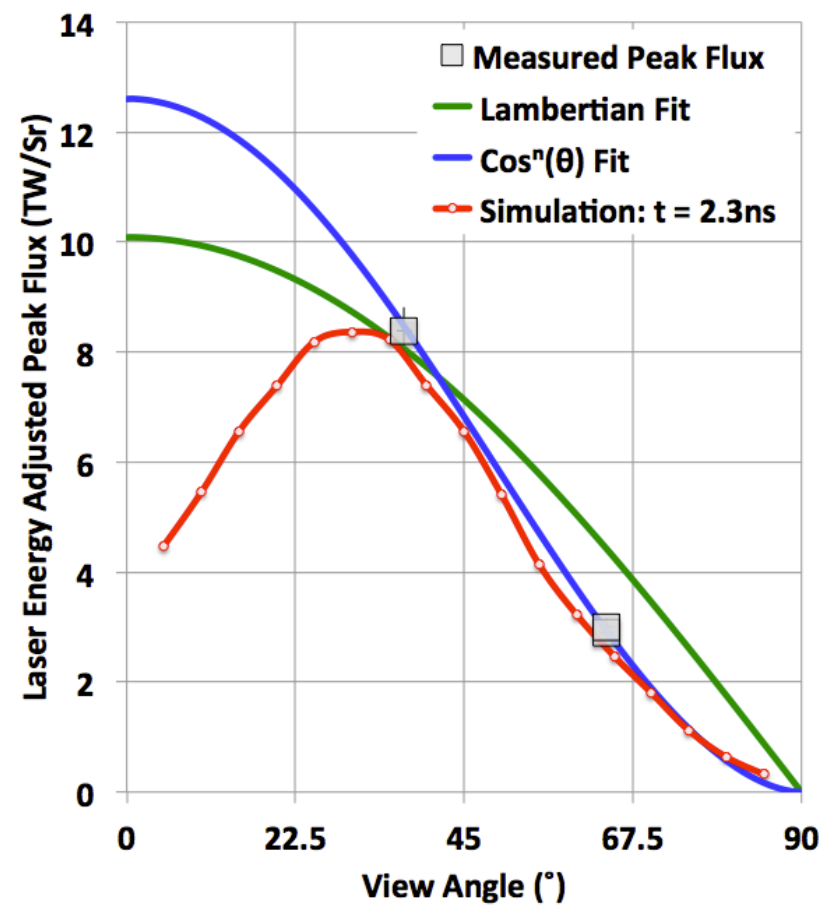

Figure 4: Peak flux measured, occurring at $t=2.3 \mathrm{~ns}$, measured at $37^{\circ}$ and $64^{\circ}$ to the hohlraum axis: blue $-\cos ^{n}(\theta)$ fit, green - Lambertian fit $(n=1)$, red - NYM simulation at time of peak emission.
Accurate measurement of the uniformity and density of the foam is critical to benchmark simulations so multiple methods were used for material characterization: (1) gravimetric, (2) low spatial resolution, x-ray radiography at 8.6 $\operatorname{keV}(\mathrm{Zn} \mathrm{K}-\alpha)$, (3) High energy ( 15 keV) high-resolution, radiography and (4) high-resolution monochromatic radiography at $5.4 \mathrm{keV}(\mathrm{Cr} \mathrm{K}-\alpha)$ [32]. Measurements (1) and (4) provide independent verification of the foam density and agree to $\pm 2.3 \%$. Knowing the elemental composition is necessary to correctly predict the foam opacity. Combustion analysis from multiple batches indicate that by mass this was: $69.6 \pm 0.2 \% \mathrm{C}, 5.4 \pm 0.1 \% \mathrm{H}$, and $24.2 \pm 0.1 \% \mathrm{Cl}$. Trace amounts $(<0.3 \%)$ of $\mathrm{N}$ and $\mathrm{S}$ were present.

\subsection{Half-hohlraum x-ray drive results}

Initial experiments characterized the x-ray drive that will enter the foam cylinder by removing the tube and leaving only the $\mathrm{SiO}_{2}$ aerogel disc - N110622 - shown in figure 2(a). Measurements were taken with the soft x-ray power diagnostic Dante [33]. The NIF has an upper and lower Dante diagnostic at $(\theta-\phi)$ of $\left(64^{\circ}-350^{\circ}\right)$ and $\left(143^{\circ}-274^{\circ}\right)$. The x-ray flux emitted through the $\mathrm{SiO}_{2}$ aerogel was measured at $64^{\circ}$ on shot N110622 and using the upper Dante and on a separate shot - N110628 - at $37^{\circ}$ by physically rotating the target $180^{\circ}$ within the NIF target chamber and repeating the experiment using the upper hemisphere laser beams and the lower Dante - shown in figure 2(b). Simulations using the radiation hydrodynamics code NYM are minimally tuned to match these result using multipliers between 0.9 and 1.0 on the Au opacity used in the hohlraum calculation, and as shown by the green lines in figure 3 provide a good match to the measured flux leaving the hohlraum through the $\mathrm{SiO}_{2}$ aerogel disc [34]. Since only the $\mathrm{x}$-ray flux entering the foam cylinder is important for the Marshak wave investigation we don't further discuss the implications of tuning the Au opacity in the hohlraum calculations but rather assert that armed with simulations that match the temporal history and absolute quantitative fluxes measured within the measurement errors is sufficient and indeed necessary to accurately characterize the Marshak wave behavior.

To compare with analytical models we assert that the hohlraum be described by a single temperature. Typically the angular distribution of emission is assumed to be Lambertian $(\propto \cos (\theta))$, but figure 4 demonstrates that the two results are inconsistent with this, so instead a $\cos ^{n}(\theta)$ dependence is fitted to the angular distribution. We find a best fit to the results with $n=1.5$ which results in a peak radiation temperature through the m-band absorber of $307 \pm 5 \mathrm{eV}$ for a laser energy of $360 \pm 2.5 \mathrm{~kJ}$. The angular distribution $\theta>37^{\circ}$ is well-matched by the NYM simulations, but deviates at small angles where the simulations indicate that the flux is reduced compared to a Lambertion due to the viewfactor through the opposite hohlraum LEH. The difference between simulation and the $\cos ^{1.5}(\theta)$ fit for $\theta<37^{\circ}$ results in only a $3 \%$ change in radiation temperature. 
It is important to note that when the foam cylinder is added to the target, shown in figure 2(c), the temperature history at the plane between the $\mathrm{SiO}_{2}$ aerogel disc and foam cylinder, indicated by the blue arrows, is different to that shown by the green lines in figure 3. This is due to the albedo provided by the foam cylinder which, once heated, emits radiation back towards the hohlraum. This cannot be measured directly when the foam cylinder is in place and so is extracted from the simulations and is shown by the blue dashed line in 3 for the supersonic Marshak case to be discussed later N121108. It should be noted that the slight difference in the rising gradient of the $\mathrm{x}$-ray flux between the two simulations is a result of small change to the shape of the laser pulse - for N110628 the laser pulse was flat-topped, but for N121108 and N120912, a $20 \%$ slope was added while maintaining the same total energy. For the $367.9 \mathrm{~kJ}$ of laser energy delivered to the hohlraum on shot N121108, the simulation of the hohlraum using the tuned Au opacity multipliers used to match the experimental data from N110628 reaches a peak temperature of $327 \mathrm{eV}$. It is also important to draw attention to another effect introduced by the foam cylinder. The additional energy sink presented by the material above the $\mathrm{SiO}_{2}$ aerogel disc results in a capacitative effect which causes the temperature to rise and fall more slowly than if the foam cylinder is removed. Once the lasers have turned off at $2.3 \mathrm{~ns}$, the hohlraum is filled with Au plasma and so optically thick to thermal re-emitted radiation from the foam cylinder. The result is that the higher albedo of the foam cylinder keeps the hohlraum temperature higher for longer. Results from the NYM simulations indicate that once the laser has turned off the foam cylinder reaches an albedo of about 0.73 , which if used in a simple energybalance calculation indicates that the presence of the foam cylinder results in a $12 \%$ increase in the hohlraum temperature once the laser has turned off - similar to that seen at about $3.0 \mathrm{~ns}$ in figure 3 .

\subsection{Marshak front arrival results}

Radiation flow is studied in the foam cylinder with the upper Dante used to quantify the heat wave arrival. This viewed the top of the cylinder at $64^{\circ}$ and was configured to measure x-ray energies between $50-1000 \mathrm{eV}$ in ten different energy bands. The results of unfolding the x-ray flux vs. time from the ten measurements is shown for the Cl-doped foam in figure 5 and $\mathrm{SiO}_{2}$ aerogel in figure 6. An early-time $(t<3.0 \mathrm{~ns})$ x-ray background signal is attributed to unblocked $\mathrm{x}$-rays emitted by the hohlraum and is plotted for the subsonic case (N120912). This background is removed from the other lines in figures 5 and 6 . A steep rise in signal due to the front arrival is attributed to the Marshak wave arrival. When the laser energy is decreased and density increased the front is seen to stall and become subsonic - N120912 - producing no steep rise in x-ray flux. This signal was used to background-correct the other data for comparison with simulations.

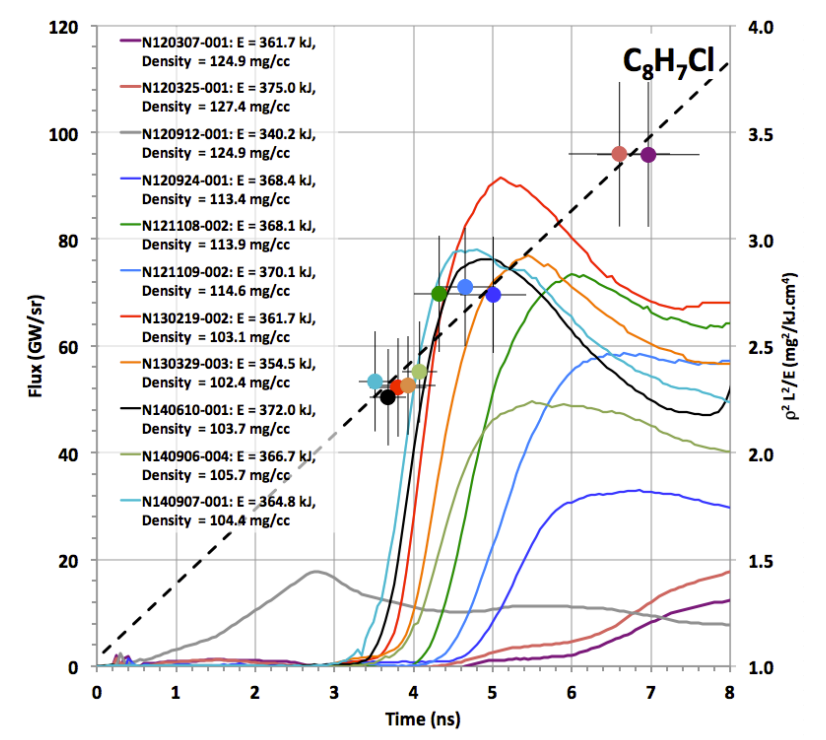

Figure 5: X-ray flux measurement of Marshak wave arrival in chlorine-doped polystyrene foam. Right-hand axis is the ratio of $\rho^{2} L^{2}$ and laser energy (E) plotted against 8.0 GW/Sr arrival time. The time-dependent background x-ray flux - N120912 - shown in gray was subtracted from shots prior to N130219.

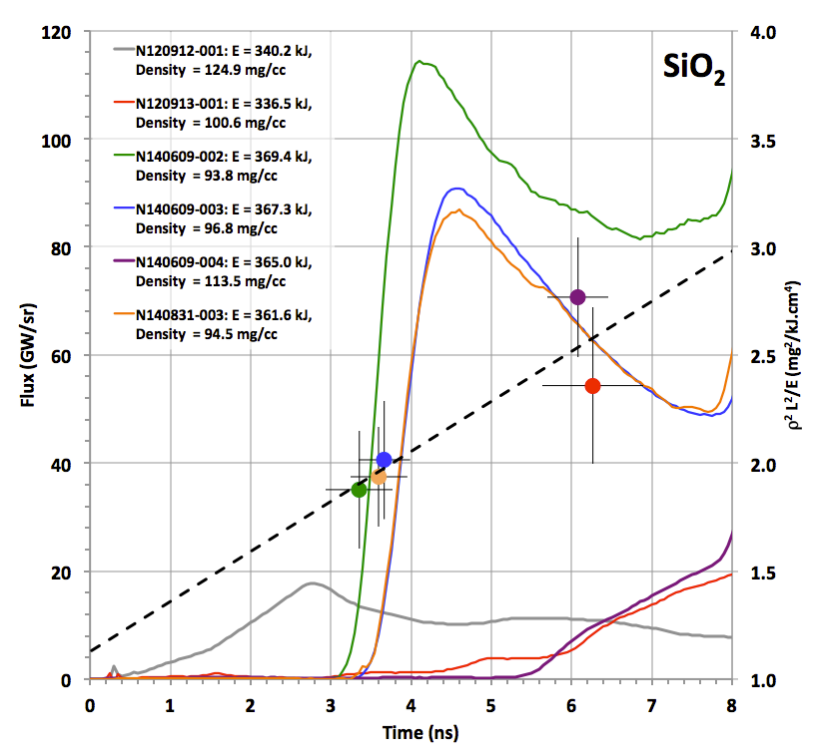

Figure 6: X-ray flux measurement of the Marshak wave arrival in silicon dioxide aerogel. Right-hand axis is the ratio of $\rho^{2} L^{2}$ and laser energy (E) plotted against $8.0 \mathrm{GW} / \mathrm{Sr}$ arrival time. The timedependent background x-ray flux - N120912 - shown in gray was subtracted from shots prior to N140609. 
The time of arrival of $8.0 \mathrm{GW} / \mathrm{Sr}$ above background (equivalent to a temperature of $65 \mathrm{eV}$, assuming the heat front initally breaks out over $2 / 3 \mathrm{rds}$ of the $2.0 \mathrm{~mm}$ diameter foam) was compared to simulations. At these temperatures the absolute flux uncertainty is $5-7 \%$. The absolute error on this time was calculated incorporating the uncertainty in diagnostic cross-timing $(\sim 40 \mathrm{ps})$, arrival of the laser beams $(\sim 25 \mathrm{ps})$, oscilloscope sweep non-linearity $(\sim 0.8 \%)$, and temporal uncertainty associated with the absolute calibration of the background corrected flux.

This is the largest contributing error bar, being 2.0 $\mathrm{GW} / \mathrm{Sr}$ at a flux of $8.0 \mathrm{GW} / \mathrm{Sr}$, but due to the steep signal rise, only adds $\approx 150 \mathrm{ps}$ to the uncertainty. Re-casting Eq. (5) in the form $t(x)$ where $x=L=2.8 \mathrm{~mm}$ it is seen that the heat front arrival is a function of $\rho^{2} L^{2} / \sigma T^{4}$. Asserting that $\sigma T^{4} \propto$ laser energy (E) this ratio is also plotted in figures 5 and 6 . If Eq. (5) is valid, then a linear trend would be expected where the gradient is inversely related to the integrated opacity and internal energy of the cylinder material. A linear fit to the data for Cl-doped foam has a reduced- $\chi^{2}=2.3$, indicating good agreement with the simple $1 \mathrm{D}$ scaling, which assumes a constant temperature drive, and $\kappa$ and $\varepsilon$ parameterized independent of $\rho$. Since only the gradient is of concern, the $1 \sigma$ errors assume only a statistical uncertainty in laser energy of 2.5 $\mathrm{kJ}$ and in density of $2.3 \%-3 \sigma$ error bars are shown in the figures. This good fit for both $\mathrm{Cl}$-doped foam and $\mathrm{SiO}_{2}$ aerogel provides good evidence that the flow is diffusive and supersonic. It is also clear when comparing the linear fits that the gradients are significantly different due to the different opacity and internal energy of the materials.

We assess the progression of the front as a function of time by soft x-ray, time-gated images of the foam selfemission through the $150 \mu \mathrm{m}$ slot [35]. The spectral response of the images was restricted to a near-Gaussian centred at $460 \mathrm{eV}$ and $650 \mathrm{eV}$ with $\sigma$ of 38 and $55 \mathrm{eV}$, by combination of a $3^{\circ}$ grazing-incidence $\mathrm{Al}$ mirror and a $2.0 \mu \mathrm{m} \mathrm{V}$ filter, and $3^{\circ} \mathrm{Ni}$ mirror with a $2.0 \mu \mathrm{m}$ Fe filter. Combined with a $1.25 \mathrm{x}$ pinhole-camera and gated $\mathrm{x}$-ray detector this formed an image with $76 \mu \mathrm{m}$ resolution.

Since the Rosseland mean free path of the heated foam is $\approx 300-350 \mu \mathrm{m}$, which is less than the tube radius, the centre of the front is unable to heat the tube. Therefore the tube is heated only by the adjacent material resulting in a localized energy loss that causes the edges of the front to lag behind the center. This is important because data in figure 7 are measurements of the front position at the outer radius of the foam cylinder close to the tube.

\section{Discussion of experimental, analytic and numer- ical results}

The conditions reached in the experiments demonstrating the arrival of a super and subsonic radiation wave are shown in Table 1 and the evolution of the heat front in figure 7 . It is clear from the figure that neither case initially follows the expected $x_{f} \propto t^{\frac{1}{2}}$. Indeed for $t<3.0 \mathrm{~ns}$ the evolution is better described by $x \propto t$. There are two possible explanations for this: (1) the time required for the Marshak wave to form, and (2) the finite rise-time of the temperature drive. The inequality in Eq. (9) addresses the first of these. For foam at $150-250 \mathrm{eV}$ and $\approx 0.11 \mathrm{~g} / \mathrm{cc}$ equates to 1.3-2.5 ns, before which time Eq. (5) and the 2D Hurricane et al. model which builds on it are not valid.

The effect of the finite rise-time of the x-ray drive on the Marshak wave evolution is examined by applying the NYM simulated $T_{r}(t)$ for N121108 - dotted blue line in figure 3 - to the 1D diffusive model of Hammer et al.. We best-fit the foam $\varepsilon$ and $\kappa$ with a power-law according to Eq. (6) and find $\frac{1}{g}=3135, \alpha=4.6, \lambda=0.31$, and $f=10.8, \beta=0.98, \mu \stackrel{g}{=} 0.05$. Inserting these parameters into Eq. (7) significantly over-predicts the position of the Marshak wave, so a 0.71 multiplier on $T_{r}(t)$ is used to best match the data. Since the data in figure 7 represents the heat front position at the edge of the tube, the solid curves in figure 7 combine the Hammer et al. model of Eq. (7) with the additional drag term predicted by Hurricane et al. - see second term in the first equation of Eq. (8) in an attempt to correct for the radius of curvature. Even with this additional drag term, it is not especially surprising that a reduction in $T_{r}$ is required since we would not expect the 1D model, even with 2D cartesian corrections applied to it, to correctly predict the losses of the $2 \mathrm{D}$ cylindrical system in the experiment. This scaled model does however correctly predict the velocity and deceleration indicating that flow is diffusion dominated and that the initial evolution $x \propto t$ observed is primarily attributable to the temporal shape of the temperature source rather than a formation time related to the Milne condition.

The 2D cartesian Hurricane et al. model clearly lacks the larger cylindrical loss of the experiment and $T_{r}(t)$ dependence of Hammer et al., but can still be fitted to the supersonic radiation wave data to estimate the curvature of the heat front due to energy transport into the Au tube. We fit the data by again using a temperature multiplier and also the Au tube albedo as free parameters and calculate the front position at the edge and centre of the tube. The best fit using a temperature multiplier of 0.60 and a wall albedo - $a$ in Eq. (8) - of 0.83 are shown as grey dashed lines in figure 7 . The weak dependence on albedo means that while the temperature multiplier can be fit to a confidence of \pm 0.005 , the uncertainty in the albedo is \pm 0.05 .

Using the fit to examine the front curvature from Eq. (8) we find $R_{c}=2.1 \mathrm{~mm}$, which corresponds to the edge trailing behind the centre by only $250 \mu \mathrm{m}$ at the end of the tube. This is in good agreement with the data and adds confidence to the assertion that energy loss to the Au tube is not significant. Also in figure 7 are the results from a NYM simulation with a 288 multi-group opacity table calculated using the CASSANDRA code and internal energy from SESAME [36, 37]. The green points represent two simulations: light green assumes that $\varepsilon$ and $\kappa_{R}$ used in the simulations are correct, the dark green increases $\varepsilon$ by 

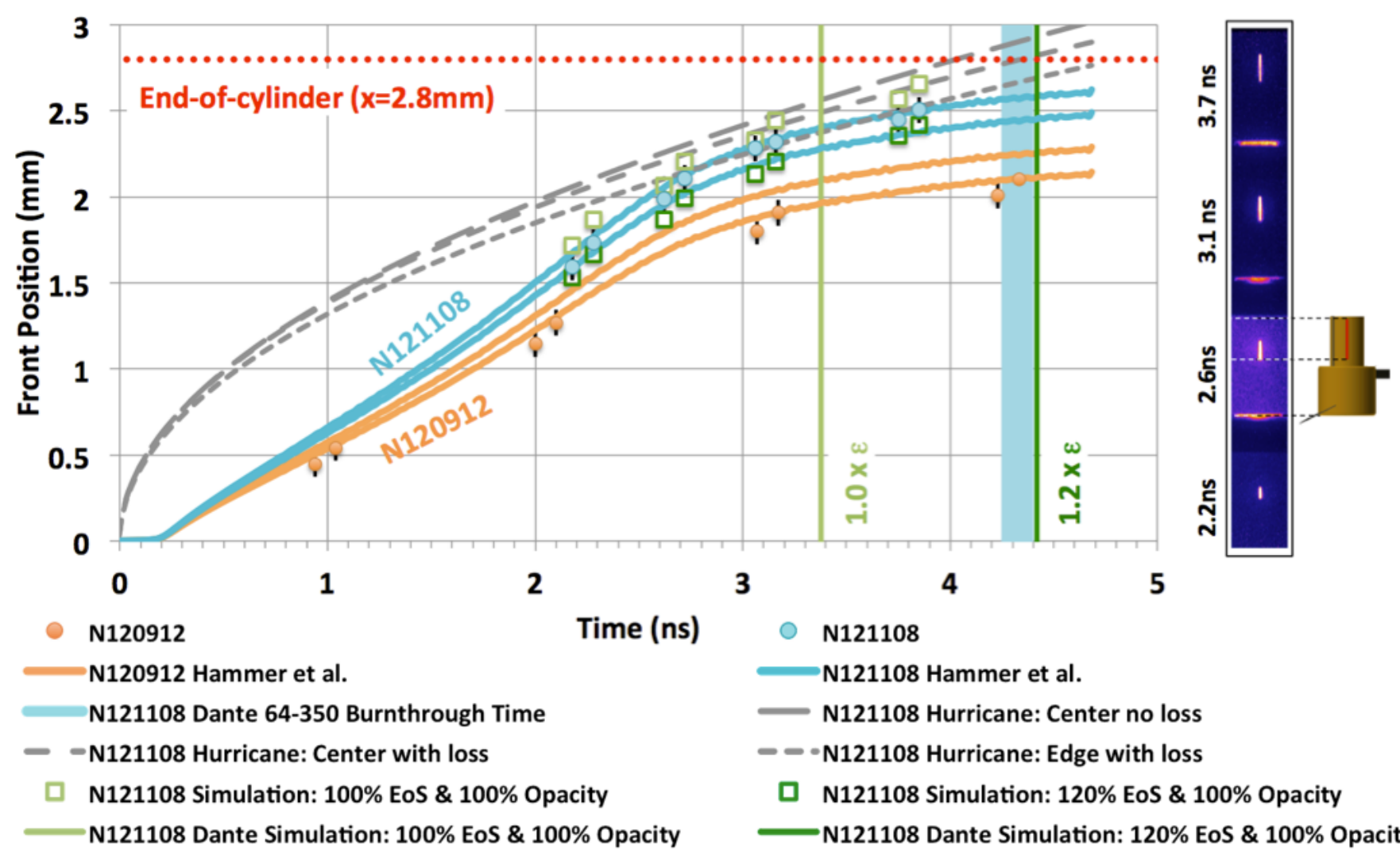

Figure 7: Marshak wave propagation data at discrete time intervals for super- and subsonic arrival. Solid lines show the Hammer et al. model corresponding to the drive temperature increased and decreased by an amount corresponding to the $\pm 2.5 \mathrm{~kJ}$ relative uncertainty in the laser energy measured and foam density increased and decreased by $\pm 2.3 \%$ to bound the solutions. The 2D Hurricane et al. model fit to the edge with loss is shown (small dashed grey) with the same fit evaluated at the tube center with and without loss (medium and large dashed grey). Simulation results with nominal and adjusted $\varepsilon$ are shown as light and dark green boxes. Vertical lines are the simulated (green) and measured (blue) upper Dante arrival times for the supersonic case; the line width is representative of the $\pm 1 \sigma$ error. Raw image data is to the right.

Table 1: Conditions for super and sub-sonic radiation wave arrival. Error bars are relative $\pm 1 \sigma$.

\begin{tabular}{lccccccc}
\hline & Shot & $\begin{array}{c}\text { Laser } \\
\text { Energy }(\mathrm{kJ})\end{array}$ & $\begin{array}{c}\text { Foam } \\
\text { Density }(\mathrm{g} / \mathrm{cc})\end{array}$ & $\begin{array}{c}\text { Peak } \mathrm{T}_{r} \text { (in) } \\
(\mathrm{eV})\end{array}$ & $\begin{array}{c}\text { Peak } \mathrm{T}_{r} \text { (out) } \\
(\mathrm{eV})\end{array}$ & $\mathcal{M}^{a}$ & \multirow{2}{*}{$\mathcal{M}^{b}$} \\
\hline Subsonic Arrival & $\mathrm{N} 120912$ & $340.2 \pm 2.5$ & $0.122 \pm 0.004$ & $320 \pm 4$ & $70 \pm 2$ & $4.3 \pm 0.4$ & $1.1 \pm 0.4$ \\
Supersonic Arrival & $\mathrm{N} 121108$ & $367.9 \pm 2.5$ & $0.114 \pm 0.003$ & $327 \pm 3$ & $118 \pm 3$ & $6.0 \pm 0.4$ & $3.8 \pm 0.4$ \\
\hline${ }^{a}$ even
\end{tabular}

\footnotetext{
${ }^{a}$ evaluated in the range $0.5<z<1.0 \mathrm{~mm}$
}

${ }^{b}$ evaluated in the range $2.0<z<2.5 \mathrm{~mm}$ 

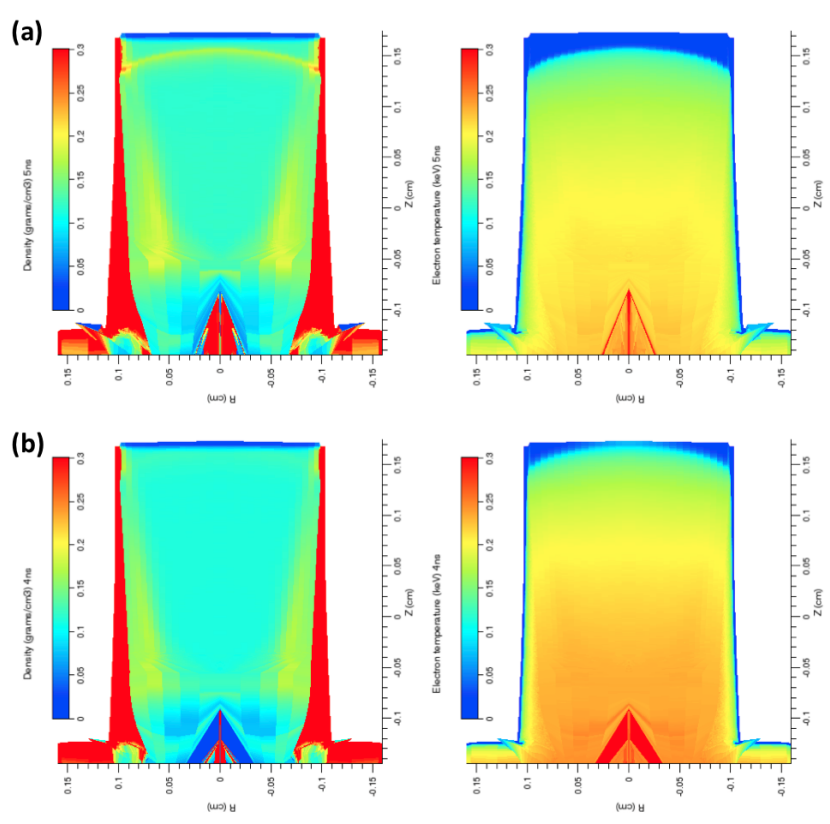

Figure 8: Density and electron temperature from NYM simulations of (a) Subsonic arrival at $t=7.0 \mathrm{~ns}$ and (b) Supersonic arrival at $t=4.3 \mathrm{~ns}$.

$20 \%$. Both the light and dark green simulations agree with the measurement within the error bars. However, we also compare the simulations to the arrival time measured by upper Dante. The $8.0 \mathrm{GW} / \mathrm{Sr}$ supersonic radiation wave arrival time is represented as a blue vertical bar which is only in agreement with the measurement when $\varepsilon$ is increased. While this is quite a significant change to $\varepsilon$, it is clear from Eq. (5) these measurements can only constrain a combination of the $\varepsilon$ and $\kappa_{R}$, so a smaller change to $\varepsilon$ is expected if $\kappa_{R}$ were also adjusted. A parametric study to best fit multipliers on both the opacity and specific internal energy to all the data is the subject of a separate publication [21].

Figure 8 shows the simulated density and electron temperature just prior to burnthrough for the super and subsonic radiation wave both with the $20 \%$ increase in $\varepsilon$. The electron temperature plot illustrates the minimal curvature of the front and is in good agreement with the Hurricane et al. estimate. The simulated density in figure 8(a) shows how the higher average density and lower laser energy causes the Marshak wave to stall and becomes subsonic producing a curved shock, while in figure $8(\mathrm{~b})$ there is no densification at the location of the front indicating that the Marshak wave is supersonic.

For the supersonic case in table 1, different numerical approaches were tested using the radiation hydrodynamics code CORVUS [38]. For increasing $n$ the $S_{n}$ method will reach the exact solution while since IMC is a statistical approach it should reach the same solution, but is not rigorously 'exact'. The same $T_{r}(t)$ shown in blue in figure 3 that was extracted from NYM and used in the analytical models was applied in as a spatially uniform source to the bottom surface of the a Cl-doped cylinder and $\mathrm{Au}$ tube. The same 288 multi-group opacity from CASSANDRA and equation of state data from SESAME that was used in the NYM simulations was applied with multipliers on each derived from the best fit of NYM simulations to the data. The CORVUS simulations were run using a diffusion model, IMC and $S_{4}, S_{8}$ and $S_{16}$. The IMC method was implemented in the same way as in the NYM simulations earlier. Up to $8 \times 10^{5}$ 'photon' particles are sourced per time step, which results in around $1 \times 10^{8}$ particles surviving in total. The results were tested for convergence by adding more particles, and this did not change the result; the results are shown in figure 9 .

Clear differences are apparent between the diffusion model and other more complex approximations, while the difference between IMC and $S_{n}$ simulations are more subtle. The diffusion approximation functions poorly around high spatial gradients in the radiation energy density. Therefore, with a standard diffusion scheme with a form following Ficks law [19], the imposed boundary temperature will overestimate the energy sourced into the problem and the Marshak wave will arrive earlier. CORVUS employs a Robin boundary condition which is more consistent with the transport solution and guarantees that the energy deposited is bounded by the amount of energy incident on the boundary, allowing a good match at early time. However, there is no such correction further down the tube. Here, near the Marshak front, the local gradient in the radiation energy density is a poor description of the flux. This leads to diffusion overestimating the flow of energy down the tube and an earlier arrival time. Neither the $S_{n}$ or IMC methods encounter this issue.

Comparison of the IMC and $S_{16}$ results show that the time at which the top of the foam cylinder reaches $100 \mathrm{eV}$ is within 0.1 ns or $2.5 \%$ which is the approximate uncertainty in the simulations. The $S_{n}$ results show convergence with only a slight difference between the $S_{8}$ and $S_{16}$ results. In the $S_{4}$ simulation the limitation of a small number of 'rays' (only three per quadrant for $S_{4}$ ) is clear from both the more pronounced curvature of the heat front when it reaches the end of the tube, and also the heat front evolution in figure $9(\mathrm{~b})$. Initially the position of the heat front lags behind the IMC simulations indicative of the diffusion model due to the poor angular resolution. With each decreasing order of $S_{n}$ the same energy is sourced over fewer angles, for $S_{4}$ each ray is approximately $45^{\circ}$. With fewer angles more energy is sourced in the forward direction and also more into the Au tube leading to a faster propagation early in time. However as the heat front propagates the heating of the Au tube directly from the source decreases until the propagation is primarily dependent upon foam emission. In the $S_{4}$ case this will be far slower because with fewer rays the length of Au tube heated directly by the source is smaller causing the reversal seen at $2.3 \mathrm{~ns}$ in 9(b). Quite remarkably, the 1D analytical model that is a single-parameter fit to the data, is within $0.1 \mathrm{~mm}$ of all the numerical solutions throughout the evolution of the 

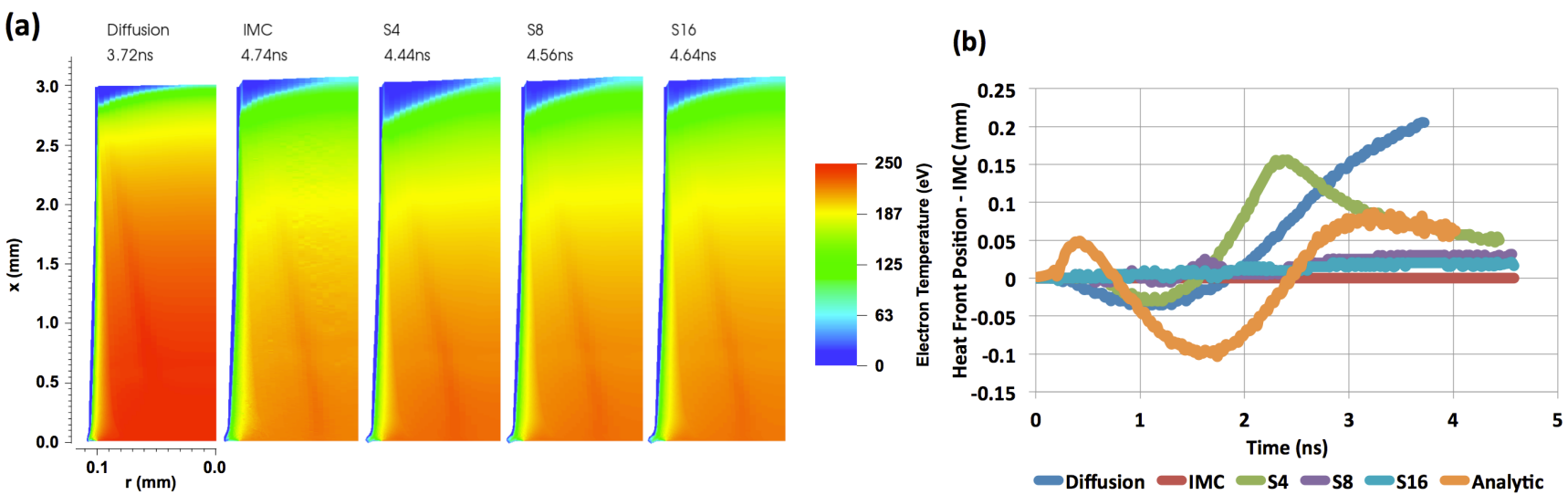

Figure 9: Comparison of simulations using the diffusion, IMC and $S_{n}$ approximations to radiation transport in the radiation-hydrodynamics code CORVUS. (a) Comparison of the electron temperature is shown at different times corresponding to when the centre $(r=0.0 \mathrm{~mm})$ of the top of the cylinder reaches an electron temperature of $100 \mathrm{eV}$. (b) The position of the heat front at the centre of the tube as a function of time for each method relative to the IMC simulation. The analytic model is that of Hammer et al. that was fit to the data in figure 7 .

heat front. It also shows some similar traits to the numerical simulations lagging behind the IMC simulation initially, but then overshooting similar to the $S_{4}$ simulation at about $2.5 \mathrm{~ns}$.

Interestingly there is still a slight difference in both the temperature profile and arrival time between the IMC and $S_{16}$ results, but this is of order the cell-size used in the simulations $(5 \mu \mathrm{m})$. The electron temperature at the end of the foam reaches $100 \mathrm{eV}$ in the IMC and $S_{16}$ simulations at 4.74 and $4.64 \mathrm{~ns}$ respectively. This is in reasonably good agreement with the measured time at which the Marshak front reaches $100 \mathrm{eV}$ of $4.61 \pm 0.11 \mathrm{~ns}$, but the heat front curvature means that the source size varies with time, and so the area-averaged radiation temperature cannot be directly compared with the simulated electron temperature at the centre of the tube. Measurements of the heat front curvature would be required to better constrain the simulations.

\section{Conclusions}

To conclude, we have demonstrated the production of a supersonic diffusive radiation wave in a previously inaccessible regime. Measurement of the absolute flux emitted as the front reaches the end of a foam sample and images of the front position at multiple times better constrain the radiation flow than in any prior work. Application of two diffusion-based analytical models do not agree in absolute terms with the data due to their limited 1D and 2D cartesian scope. However, simple scaling of the Hammer et al. model produces very good phenomenological agreement. Simulations employing a simple diffusion approximation grossly overestimate the energy entering the problem due to the necessary boundary conditions applied. Higher accuracy $S_{n}$ with $n>4$ and IMC transport approximations are in good agreement with the measured data provided that $\varepsilon$ is increased by $\approx 20 \%$, and provide quantitative validation of the conditions under which the front transitions from super- to subsonic. As such the results provide a constraining measurement of the foam material properties in addition to testing the radiation transport methods applied.

We are grateful to the dedication of the NIF operation team. This work was supported by UK Ministry of Defence and performed under the auspices of the US Department of Energy by Los Alamos National Laboratory under contract DE-AC52-006NA25396 and Lawrence Livermore National Laboratory under contract DE-AC52-07NA273444. British Crown Copyright (C) 2015.

[1] D. Mihalas 2003 ASP Conference Series. 288, 459

[2] R. P. Drake. 2006 Chapter 7: Radiation Hydrodynamics in HighEnergy-Density Physics. Springer

[3] J. Lindl. 1995 Physics of Plasmas 2, 3933

[4] R. E. Marshak. 1958 Physics of Fluids 1, 24

[5] B. Su, G. L. Olsen. 1996 J. Quant. Spectrosc. Radiat. Transfer 56,337

[6] G. C. Pomraning. 1979 J. Quant. Spectrosc. Radiat. Transfer 21, 249

[7] E. W. Larsen, G. C. Pomraning. 1980 SIAM Journal on Applied Mathematics, 39, 201

[8] L. Ensman. 1994 Astrophysical Journal, 424, 275

[9] E. I. Moses, R. N. Boyd, B. A. Remington, C. J. Keane, R. AlAyat. 2009 Physics of Plasmas 16, 041006

[10] T. Afshar-rad, M. Desselberger, M. Dunne, J. Edwards, J. M. Foster, D. Hoarty, M. W. Jones, S. J. Rose, P. A. Rosen, R. Taylor, and O. Willi 1994 Physical Review Letters, 73, 74

[11] O. Willi, L. Barringer, C. Vickers, and D. Hoarty. 2000 Astrophysical Journal Supplement Series 127, 527

[12] D. Hoarty, A. Iwase, C. Meyer, J. Edwards, O. Willi. 1997 Physical Review Letters 78, 3322

[13] J. C. Bozier, G. Thiell, J. P. Le Breton,S. Azra, M. Decroisette, D. Schirmann. 1986 Physical Review Letters 57, 1304

[14] C. A. Back, J. D. Bauer, J. H. Hammer, B. F. Lasinski, R. E. Turner, P. W. Rambo, O. L. Landen, L. J. Suter, M. D. Rosen, W. W. Hsing. 2000 Physics of Plasmas 7, 2126

[15] F. Graziani. 2000 Computational Methods in Transport: Verification and Validation, Chap I. Springer-Verlag Berlin Heidelberg

[16] Y. B. Zel'dovich and Y. P. Raizer (1966) II. \$10. in Physics of Shock Waves and High-Temperature Hydrodynamic Phenomena. Dover Publications Inc., NY

[17] G. C. Pomraning. 1973 The Eqs. of Radiation Hydrodynamics, 
Chap III. Pergamon Press, Oxford

[18] J. I. Castor. 2004 Radiation Hydrodynamics Cambridge University Press

[19] A. Fick. 1855 Poggendorffs Annalen. 94, 59

20] A. Fick 1995 Journal of Membrane Science 100 33-38

[21] T. M. Guymer et al. 2014 submitted to Physics of Plasmas

[22] J. Hammer, M. D. Rosen. 2003 Physics of Plasmas 10, 1829.

[23] M. M. Marinak, B. A. Remington, S. V. Weber, R. E. Tipton, S. W. Haan, K. S. Budil, O. L. Landen, J. D. Kilkenny, R. Wallace. 1995 Physical Review Letters 75, 3677

[24] O. A. Hurricane, J. Hammer. 2006 Physics of Plasmas 13113303

[25] E. A. Milne. 1921, Mon. Notices Roy. Astron. Soc. 81361375

[26] T. A. Brunner. 2002 OSTI ID: 800993, SAND2002-1778

[27] S. Chandrasekhar. 1960 Radiative Transfer, Dover Publications Inc., NY

[28] K. D. Lathrop. 1968 Nucl. Sci. Eng. 32, 357

[29] J. A. Fleck, J. D. Cummings. 1971 J. Comput. Phys. 8, 313

[30] A. S. Moore, J. Morton, T. Guymer, N. Bazin, C. Bentley, M. Stevenson, J. L. Kline, P. Keiter, M. Taccetti, K. Mussack, B. Peterson, D. W. Schmidt, C. Hamilton, N. Lanier, J. Workman. 2012 Fusion Science and Tech. 63, 76

[31] J. M. Williams, A. J. Gray, M. H. Wilkerson. 1990 Langmuir 6, 437

[32] N. E. Lanier, C. Hamilton, J. M. Taccetti. 2012 Review of Scientific Instruments 83, 10E521

[33] E. L. Dewald, K. M. Campbell, R. E. Turner, J. P. Holder, O. L. Landen, S. H. Glenzer, R. L. Kauffman, L. J. Suter, M. Landon, M. Rhodes, D. Lee. 2004 Review of Scientific Instruments 75, 3759

[34] P. D. Roberts, S. J. Rose, P. C. Thompson, R. J. Wright. 1980 Journal of Physics D 13, 1957

[35] F. Ze, R. L. Kauffman, J. D. Kilkenny, J. Wielwald, P. M. Bell, R. Hanks, J. Stewart, D. Dean, J. Bower, R. Wallace Review of Scientific Instruments, 63, 5124 (1992)

[36] B. Crowley, J. Harris. 2001 J. Quant. Spectrosc. Radiat. Transfer 71,257

[37] B. I. Bennet, J. D. Johnson, G. I. Kerley, G. T. Rood. 1978 OSTI ID: 5150206

[38] A. J. Barlow. 2008 Int. J. Numer. Meth. Fluids 56, 953 